\title{
Biologically friendly room temperature ionic liquids and nanomaterials for the development of innovative enzymatic biosensors: part II
}

\author{
Daniele Zappi $^{a *}$, Serena Gabriele ${ }^{a}$, Lorenzo Gontrani $^{b}$, Danilo Dini $^{a}$, Claudia Sadun $^{a}$, Federico \\ Marini $^{a}$, Marta Letizia Antonelli ${ }^{a}$ \\ ${ }^{a}$ Department of Chemistry, University of Rome "La Sapienza" - p.le A. Moro, 5 - 00185 Rome - Italy \\ ${ }^{b}$ Department of Chemistry "Giacomo Ciamician”, University of Bologna, v. F. Selmi 2 - 40126 Bologna - Italy \\ *Corrresponding Author: daniele.zappi@uniromal.it
}

\begin{abstract}
A newly modified electrode based on glassy carbon (GC) has been prepared and characterized electrochemically for application in electroanalytical chemistry. In particular, a GC screen-printed electrode (SPE) has been modified with nanostructures, namely multi-walled carbon nanotubes (MWCNTs), and $\mathrm{TiO}_{2}$ nanoparticles, and combined with a new generation of eco-friendly roomtemperature ionic liquids (RTILs). The green RTILs here used are suitable for the immobilization of enzymes on the electrode surface and, additionally, facilitate the kinetics of electron transfer due to their intrinsic electrical conductivity. Upon evaluation of these newly modified electrodes we found an improvement in terms of electrochemically active area (Aea) with respect to the electrodes we previously reported. The modified SPEs were then used as substrates for the construction of two enzymatic biosensors for analytical applications: the first is an enzymatic biosensor based on alcohol dehydrogenase (ADH) for the analysis of ethyl alcohol; the second biosensor is based on lipase enzyme and has been tested for the analysis and the classification of Extra Virgin Olive Oil (EVOO). The performances of the here projected sensors appear comparable with biosensors having similar finalities. It is here envisaged that such a kind of electrodes could represent the starting tool for the construction and the definition of new portable devices for screening and field analyses.
\end{abstract}

KEYWORDS: Enzymatic biosensor; Ionic liquid; Nanostructured SPE; Alcohol dehydrogenase; Lipase; EVOO

\author{
Abbreviations: \\ GC: Glassy Carbon \\ SPE: Screen Printed Electrode \\ MWCNT: Multi-Walled Carbon Nanotube \\ $\mathrm{A}_{\mathrm{ea}}$ : Electrochemically active area \\ RTIL: Room Temperature Ionic Liquid \\ Ch: Choline \\ Phe: Phenylalanine \\ Ser: Serine \\ ADH: Alcohol dehydrogenase \\ EVOO: Extra-Virgin Olive Oil
}




\section{Introduction}

In recent years traditional analytical procedures have been implemented by integrating biological components into analytical tools like biosensors which have been the subject of numerous researches. The passage from sensors to biosensors allows a higher degree of selectivity and operational simplicity to be achieved, if compared to traditional analytical procedures. Currently, biosensors are widely used in many fields ranging from the biomedical sector [1] e.g. glucose sensors for diabetic patients or screening tests in clinical ambit, to the agri-food sector [2,3] in which biosensors are used for the determination of pesticide residues in food, for the analysis of markers in food quality evaluation and for environmental protection [4,5].

Following a previous work concerning the use of green RTILs (room temperature ionic liquid) and MWCNT (multi-walled carbon nano tubes) [6] in amperometric sensors and biosensors, the present study aims to achieve better performances of the modified WE (working electrode) for biosensing applications in the agri-food sector. The goal of this research is to construct and characterize new biosensors, useful to analyze olive oils and also alcohols in beverages, in a reliable and simple way. The biosensors that are being developed are also designed for field analyses, without the need to use laboratory equipment. These biosensors must be inexpensive, disposable, usable by non-specialists and mass-produced.

RTILs are largely employed in electrochemical researches [7-10] for their good chemical-physical properties, such as extremely low volatility, good conductivity, low chemical reactivity and thermal stability. In particular, RTILs are often used in the development of biosensors [11] thanks to their ability to entrap the bioactive units without altering their properties.

The here applied RTILs are of generation IV, i.e. based on choline [Ch], phenylalanine [Phe], and serine [Ser]. These RTILs are environmental friendly compounds [12], very different from the ionic liquids of previous generations [13-15]. To this purpose, the electrochemical performances of an SPE (screen-printed electrodes) sensor can be studied by adding another nanomaterial on the WE surface and varying the relative proportion with respect to the MWCNT. As a new nanomaterial, the titanium dioxide nanoparticles, $\mathrm{TiO}_{2}$ anatase polymorph, were chosen. To optimize the kind and quantity of experiments to be performed, a chemometric approach [16, 17] must be adopted. Different procedures to modify the WE can be established, by varying the steps and the deposition methods of RTILs and nanomaterials. Moreover, in order to realize biosensors with the best electrochemical and analytical performances, different methods of enzymatic catalysis can be investigated. To determine ethyl alcohol content in beverages a biosensor ADH (alcohol dehydrogenases) based could be used $[18,19]$. The lipase enzyme can be used to construct the proper biosensor to analyze extra virgin olive oils (EVOO) by measuring the influence of the oil antioxidant composition on the electrochemical response, with the variation of the cultivar and the geographical origin of the olive trees [20,21].

\section{Materials and methods}

\subsection{Materials}

Potassium hexacyanoferrate $(\mathrm{III})\left(\mathrm{K}_{3}\left[\mathrm{Fe}(\mathrm{CN})_{6}\right]\right)$, sodium dihydrogen phosphate $\left(\mathrm{NaH}_{2} \mathrm{PO}_{4}\right)$, sodium hydrogen phosphate $\left(\mathrm{Na}_{2} \mathrm{HPO}_{4}\right)$ were obtained from Merck (Kenilworth, NJ, USA). Potassium chloride and nitric acid were purchased from Carlo Erba (Cornaredo, MI, Italy). ethanol, sodium hydroxide and multi-walled carbon nanotubes (MWCNT) (O.D. $=(10 \pm 1) \mathrm{nm}$; I.D. $=(4.5 \pm 0.5) \mathrm{nm}$; $\mathrm{L}=3-6 \mu \mathrm{m}$ ) were supplied from Sigma-Aldrich (Buchs, Switzerland). Anatase titanium dioxide (99\% purity; $5 \mathrm{~nm}$ ) were obtained from NanoAmor Nanostructured and Amorphous Materials Inc. (Houston, TX, USA). 
2-Hydroxy-N,N,N trimethylethan-1-aminium hydroxide (Choline hydroxide) and the two amino acids (serine, phenylalanine) were purchased from Alfa Aesar (Kander, Germany). All cholineamino acid room temperature ionic liquids (RTILs): choline-serine [Ch][Ser], cholinephenylalanine [Ch][Phe] were synthesized following the titrimetric methodology reported by De Santis et al. [12]

Fungal lipase from Candida rugosa (EC 3.1.1.3, activity: $4320 \mathrm{U} \mathrm{mg}^{-1}$ ), stored at $4{ }^{\circ} \mathrm{C}$, and alcohol dehydrogenase (ADH) from Saccharomyces cerevisiae (EC 1.10.3.2, activity: $331 \mathrm{U} \mathrm{mg}^{-1}$ ), stored at $-18{ }^{\circ} \mathrm{C}$, were from Sigma-Aldrich (Buchs, Switzerland).

Olive oils were obtained from olive samples of different cultivar and geographical origin, using a standardized method described in the next paragraphs.

All the solutions have been prepared by means of high purity deionized water (Resistance $=18.2$ $\mathrm{M} \Omega \times \mathrm{cm}$ at $25^{\circ} \mathrm{C}$; TOC $<10 \mu \mathrm{g} \mathrm{L}^{-1}$ ), obtained from Millipore Direct-Q UV3 device (Molsheim, France).

\subsection{Instrumentation}

All electrochemical experiments have been performed by means of a PalmSens Electrochemical Sensor Interface (PalmSens BV Utrecht, The Netherlands), using Screen Printed electrodes (SPEs) printed on plastic support, with glassy carbon working electrode (WE), silver reference electrode (RE), and counter carbon electrode (CE) (GSI Technologies Burr Ridge, IL, USA). For oil extraction, a crusher instrument, Knife Mill GRINDOMIX GM 300, (Retsch GmbH, Haan, Germany)and an Eppendorf centrifuge (5810 R, Eppendorf International, Hamburg, Germany) have been used.

For alcoholic degree measurements a hydrostatic balance DensiMat-CE (Gibertini, Novate Milanese, MI, Italy) has been used. For analysis of olive oil: Free Fatty Acids (FFA), Peroxide Value (PV), Polyphenols a CDR-FoodLab (CDR s.r.1 - Ginestra Fiorentina - Firenze - ITALIA) instrument was employed

\subsection{Methods}

2.3.1. Olive oil extraction method

Nowadays olive oil extraction is mostly conducted in oil mills that work in a continuous cycle. The cycle includes the olives to be washed, then ground, the olive paste braked for less than an hour and then centrifuged to obtain the separation of the oil from water and pomace. The method here developed wants to mimic the industrial one.

All the olives have been harvested during the second week of November 2016 and processed within 48 hours at a temperature below $27^{\circ} \mathrm{C}$. A weighed quantity of olives after washing was ground into the crusher, and then the olive paste, braked for 30 minutes, was centrifuged to obtain the olive oil. The oil yield was about $10-15 \%$ of the olives weight, in accordance with the industrial yields that vary significantly depending on the year.

In a previous work [6] commercial and/or artisanal EVOOs were analyzed. Many parameters can affect the oil quality during the whole production chain (harvest, storage, technological processes, etc.) and it is in general impossible to establish which parameter had the most impact on commercial olive oils. In order to be able to compare samples of oil, on which this type of problem has the least impact, a laboratory method for olive oil extraction has been developed, as described in the experimental section. This extraction method proved reliable, reproducible and safe. 
The oil samples to be analyzed were obtained from olives of sure and undisputed cultivar and geographical origin, all collected in Italy during the 2016 campaign. Seven oil samples, obtained from olives grown in Rome and Frosinone provinces of Latium region and processed with the laboratory method, were characterized by the acidity measures [22]. Looking for high-quality of EVOO the variation of the acidity is very important, this range is strictly fixed by EU Regulations (1991) [23]. Extra virgin means that the olive oil acidity level is less than $0.8 \%$, expressed as a percentage of free oleic acid. Only on the basis of their acidity, all studied oil samples can be classified as EVOOs. In Table 1 the specific geographical origin, cultivar and acidity of all examined oils are listed.

\begin{tabular}{|c|c|c|}
\hline Geographical origin & Cultivar & $\begin{array}{c}\text { Acidity } \\
\text { (free oleic acid \%) }\end{array}$ \\
\hline Fiano Romano (RM) & Nostrale & 0.23 \\
\hline Fiano Romano (RM) & Frantoio & 0.20 \\
\hline Fiano Romano (RM) & Leccino & 0.19 \\
\hline Bracciano (RM) & Leccino & 0.09 \\
\hline Isola del Liri (FR) & Leccino & 0.13 \\
\hline Arpino (FR) & Moraiolo 1 & 0.15 \\
\hline Arpino (FR) & Moraiolo 2 & 0.28 \\
\hline
\end{tabular}

Table 1 Identification parameters of the examined extra-virgin olive oils (EVOOs)

\subsubsection{Electrode Modifications}

The working electrode surface was modified with nanomaterials and RTILs. A certain amount of nanomaterials: multiwalled carbon nanotubes (MWCNT) or MWCNT blended with $\mathrm{TiO}_{2}$ nanoparticles, were dispersed in RTIL aqueous solution to facilitate the deposition. The solution of the chosen RTIL was prepared with water for electroactive area $\left(\mathrm{A}_{\mathrm{ea}}\right)$ measurements or with an appropriate buffer, $\mathrm{pH}$ 7.4 PBS buffer for Lipases and pH 8.0 PBS for ADH, for enzymatic sensors assembling. $40 \mu \mathrm{L}$ of these suspensions were dropped on the working electrode and then dried in a desiccator overnight.

For biosensors assembling, the enzyme immobilization was performed by dissolving the proper enzyme in the above-described solution, a volume of $40 \mu \mathrm{L}$ of the suspension was dropped on the working electrode surface. This immobilization gives rise to the enzyme entrapment onto the electrode surface due to the strong interactions with RTIL ions. The ionic liquid is able to ensure the highest enzyme loading, preserving its native structure and biological activity [24] The modification steps were controlled by weighing the bare electrodes, the electrodes with the wet deposition and finally with the dry one. This method showed a great reproducibility for the electrodes within a batch and through different batches.

\subsubsection{Electrochemical measurements}

Commercial SPE, printed on plastic support with bare glassy carbon working electrodes (WE) were employed, the WE diameter is $4 \mathrm{~mm}$. Reference and counter electrodes were silver and graphite respectively. These electrodes have been further modified with nanostructures and different generation IV RTILs, performing all the possible combinations. The measurements for calculating 
the electrochemically active areas $\left(\mathrm{A}_{\mathrm{ea}}\right)$ by cyclic voltammetry $(\mathrm{CV})$ at variable scan rate $(5-80 \mathrm{mV}$ $\mathrm{s}^{-1}$ ) were carried out, in the presence of $1 \mathrm{mmol} \mathrm{L}^{-1} \mathrm{~K}_{3} \mathrm{Fe}(\mathrm{CN})_{6}$ in deionized water, $100 \mathrm{mmol} \mathrm{L}^{-1}$ solution of potassium chloride $(\mathrm{KCl})$ was used as electrolyte (Figure 1).

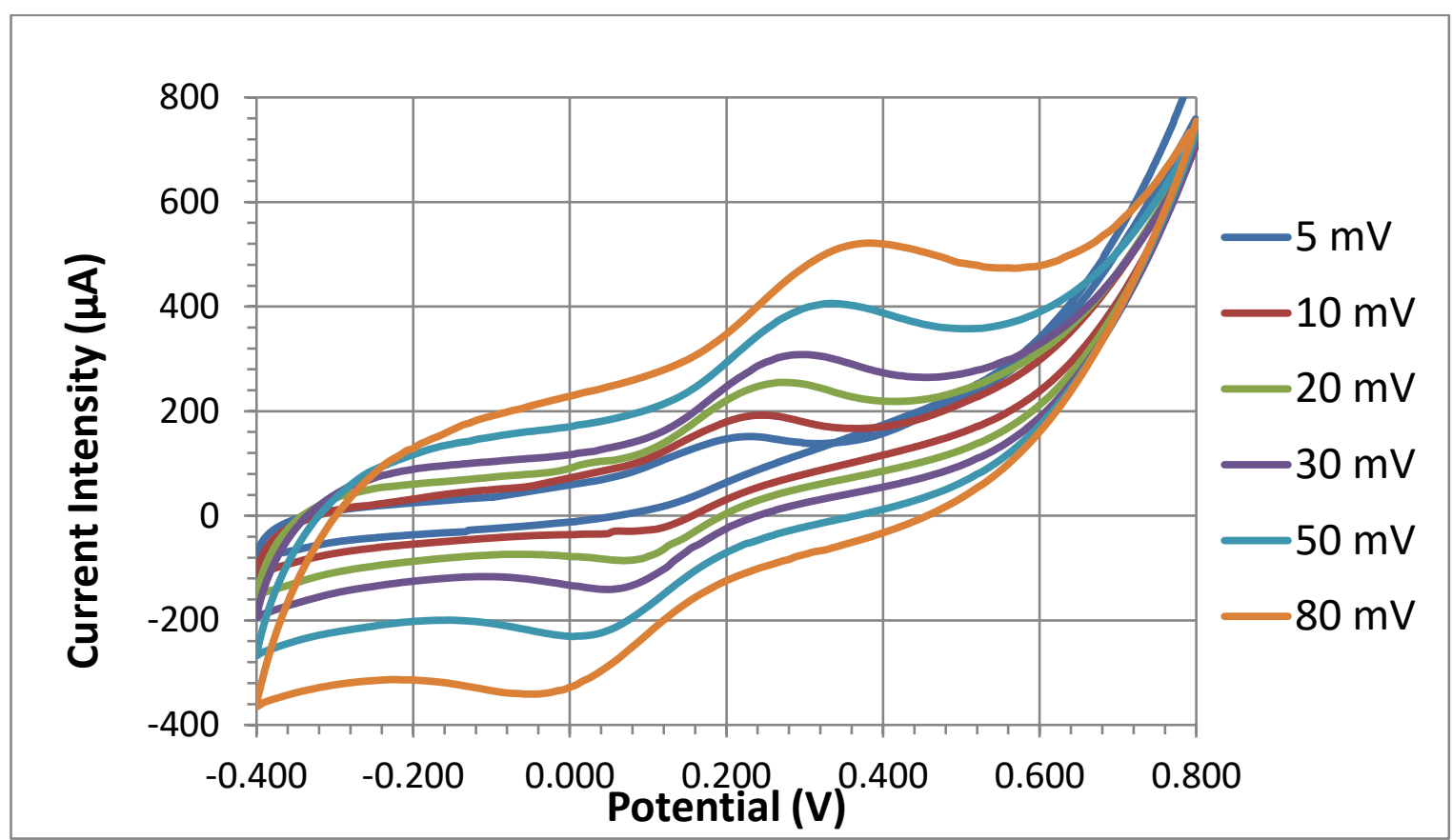

Figure 1: CV scans performed with the modified electrode GC/MWCNT/TiO $/[\mathrm{Ch}][\mathrm{Phe}]$ in presence of 1 $\mathrm{mmol} \mathrm{L}{ }^{-1} \mathrm{~K}_{3} \mathrm{Fe}(\mathrm{CN})_{6}$ in $100 \mathrm{mmol} \mathrm{L}^{-1} \mathrm{KCl}$

The electrochemically active areas have been calculated by the well-known Randles-Sevcik equation [25].

All the edible oil samples were prepared for electrochemical measurements by dissolving the oil in a $[\mathrm{Ch}][\mathrm{Ser}] /$ buffer solution with ratio oil/[Ch][Ser]/buffer 2:2:1, as reported in our preceding paper [6].

\section{Results and discussion}

\subsection{Performances of the modified working electrode}

The interactions between nanomaterials and RTILs have already been studied in a previous work [6]. The best results were obtained when the MWCNT were combined with the RTIL [Ch][Phe]. Therefore, the bare glassy carbon electrodes have been modified by drop-casting a suspension of MWCNT in [Ch][Phe] aqueous solution.

Since the $\mathrm{A}_{\mathrm{ea}}$ varies non linearly with the composition of the modified electrode surfaces, it has been investigated how the different MWCNT/[Ch][Phe] proportions could influence the performances of the platform.

In order to minimize the experiments number, an experimental design varying the levels of two independent variables $(\mathrm{N})$ has been adopted. The quantities of MWCNT (mg) and the percentage of $[\mathrm{Ch}][\mathrm{Phe}]$ in the aqueous solution were studied at three different levels, obtaining a $3^{\mathrm{N}}$ experimental design. The nine required experiments were performed in three replicates and are shown in Figure 2. The experimental results were processed with chemometric technique and the 
surface describing the effect of MWCNT and RTIL amounts on the electrochemically active surface area is shown in Figure 3.

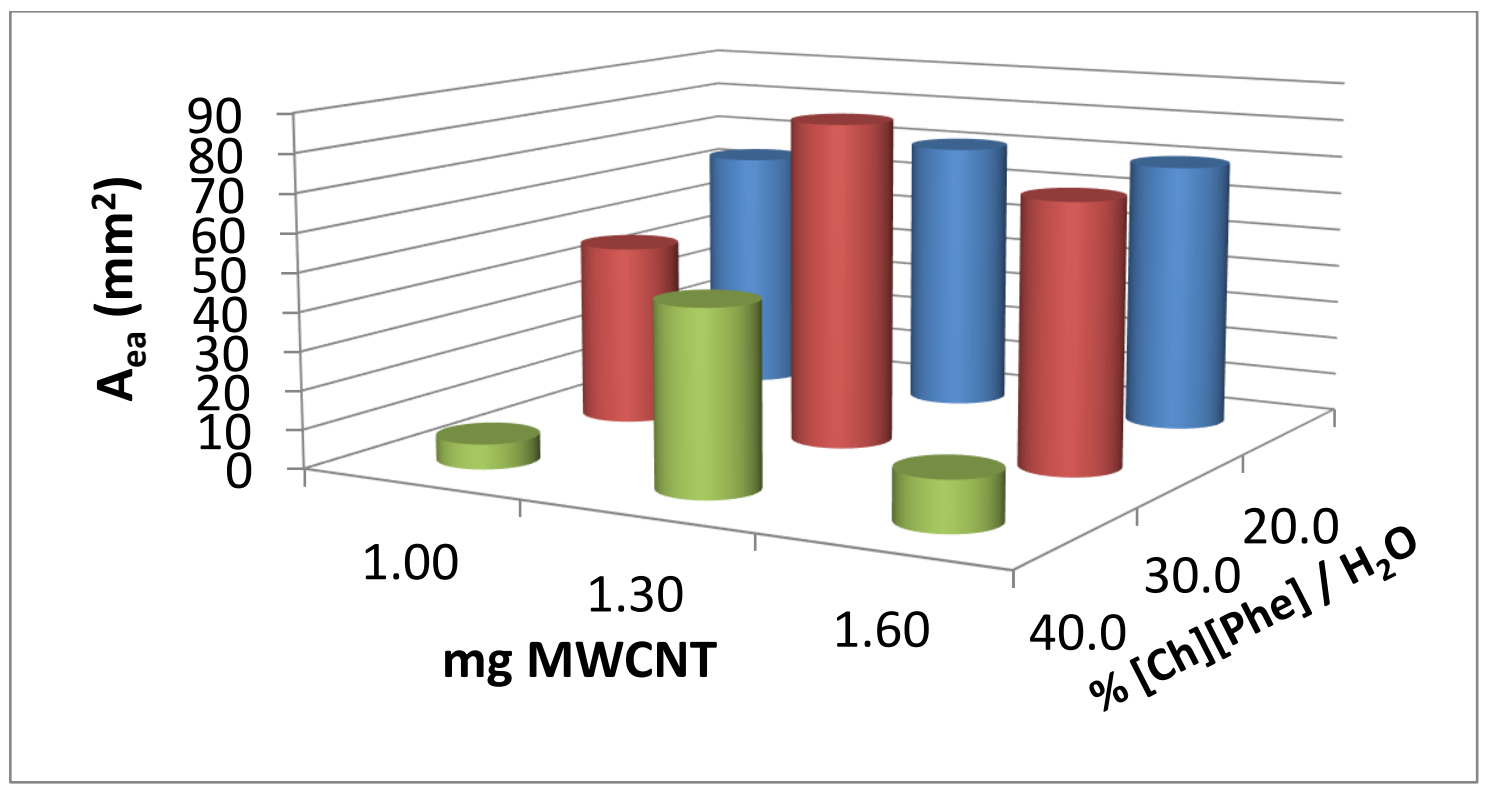

Figure 2: Effect of MWCNT amounts and [Ch][Phe]/water percentages on the electrochemically active surface area $\left(\mathrm{A}_{\mathrm{ea}}\right)$ of modified WE

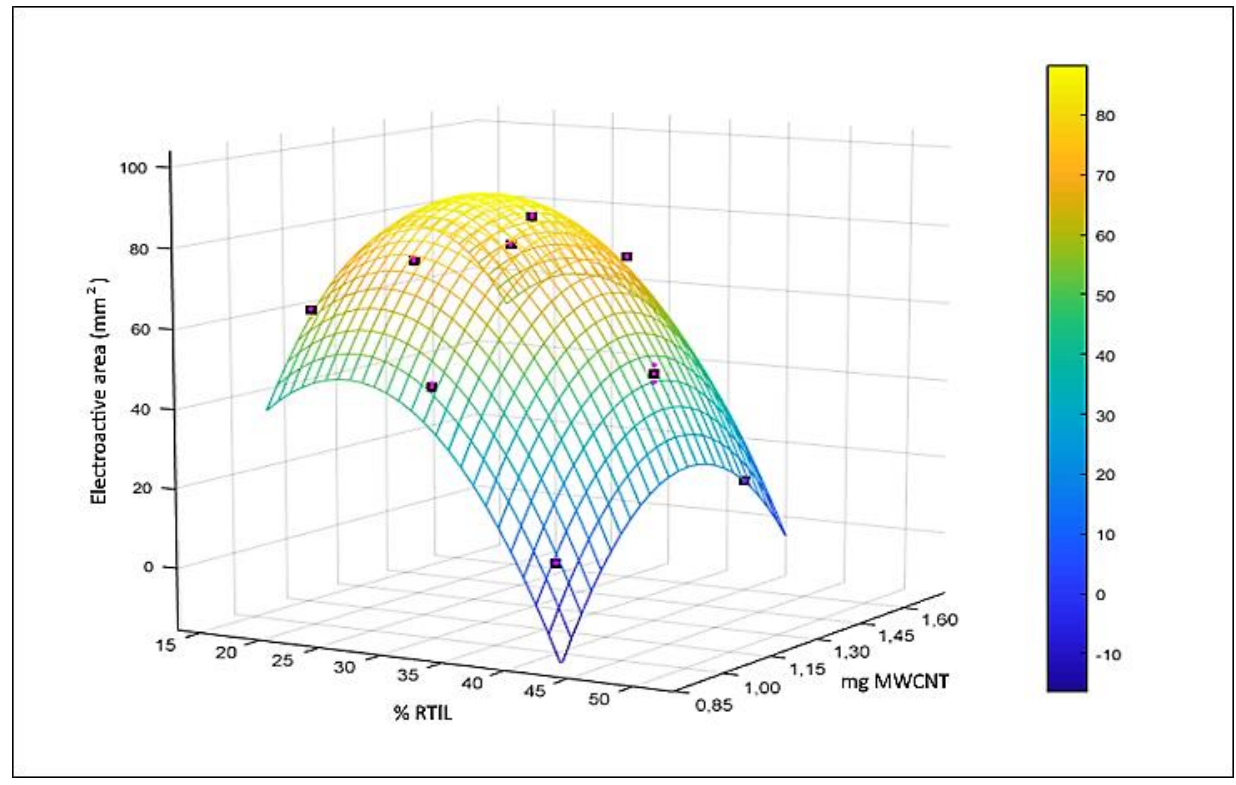

Figure 3: Response surface for the $3^{2}$ experiments according to the experimental design

The results show that the electrochemically active area is really affected by the two considered variables. In particular, the smallest electrochemically active area is obtained with a deposition containing $40 \%$ of $[\mathrm{Ch}][\mathrm{Phe}]$ in aqueous solution. This result can be attributed to an increase in mixture viscosity, due to the high RTIL [Ch][Phe] viscosity. Otherwise, using too small quantities of [Ch][Phe], good electrochemically active areas could be obtained, but MWCNT do not mix up homogeneously making problematic the deposition.

The mathematical model of the experimental design suggests that the best combination of RTIL [Ch][Phe] and the amount of MWCNT is respectively $25 \%$ and $1.3 \mathrm{mg}$ in $100 \mu \mathrm{l}$ of an aqueous solution. This combination allows obtaining an electrochemically active area of $88.009 \mathrm{~mm}^{2}$. As 
mentioned before, otherwise a \% of RTIL less than $30 \%$ does not allow homogeneous dispersions and consequently, reproducible depositions are not obtained.

The combination of $1.3 \mathrm{mg}$ MWCNT and $30 \%$ [Ch][Phe] aqueous solution had been chosen for all successive measurements. In these conditions, a small decrease in the electrochemically active area (about $4.5 \%$ ) has been observed, but a satisfactory increase in platform reproducibility has been achieved.

Knowing from the literature that interactions take place between MWCNT and other nanomaterials such as $\mathrm{TiO}_{2}$ nanoparticles [26,27], the electrochemically active areas of SPEs modified with MWCNT and $\mathrm{TiO}_{2}$ have been studied. To obtain better electrochemical performances, solid mixtures were prepared at different $\mathrm{MWCNT} / \mathrm{TiO}_{2}$ ratios, all having a final mass of $1.3 \mathrm{mg}$ as previously stated and dispersed in the $30 \%$ [Ch][Phe] aqueous solution. In Table 2 the values of the resulting areas are reported.

\begin{tabular}{|l|c|c|c|c|c|}
\hline$\% \mathrm{TiO}_{2} / \mathrm{MWCNT}$ & 5 & 10 & 30 & 50 & 100 \\
\hline $\mathrm{A}_{\mathrm{e}} \pm \mathrm{SD}\left(\mathrm{mm}^{2}\right)$ & $96.9 \pm 0.3$ & $60.82 \pm 0.03$ & $33.0 \pm 0.1$ & $34.2 \pm 0.2$ & $1.5 \pm 0.2$ \\
\hline
\end{tabular}

Table 2 Electrochemically active areas $\left(\mathrm{A}_{\mathrm{ea}}\right)$ determined for modified electrodes GC/MWCNT/TiO $/[\mathrm{Ch}][\mathrm{Phe}] /$ water. $\mathrm{TiO}_{2} \%$ is stated as the $\mathrm{TiO}_{2} / \mathrm{MWCNT}$ ratio for $1.3 \mathrm{mg}$ total mass

It is known that the hydroxyl groups on the surface of the $\mathrm{TiO}_{2}$ nanoparticles can react with the open ends of the activated MWCNT giving stable aggregates [27]. Depending on the relative concentrations of $\mathrm{MWCNT} / \mathrm{TiO}_{2}$, different types of aggregates can be formed and these can generate different surface extensions. From the obtained results it is possible to notice that a small quantity of $\mathrm{TiO}_{2}$ leads to a considerable increase in electrochemically active area, while high percentages of $\mathrm{TiO}_{2}$ do not produce a significant increase. It can be assumed that, in the last case, linear structures similar to those reported by Kang and al. can be created. On the contrary, when only $5 \%$ of $\mathrm{TiO}_{2}$ is present, it can be assumed that the surface of the $\mathrm{TiO}_{2}$ nanoparticles can be functionalized by several activated MWCNT-COOHs, improving the MWCNT distribution on the surface of the $\mathrm{TiO}_{2}$ nanoparticles. This is also evidenced by a better deposition reproducibility on the working electrode and by an excellent stability over time of the functionalized electrodes (GC/MWCNT/TiO $/[\mathrm{Ch}][\mathrm{Phe}] /$ water). In Figure 4 the electrode stabilities over time relative to the three better $\mathrm{TiO}_{2} / \mathrm{MWCNT}$ ratios are reported. Consequently to the previous measurements, the electrodes modified with a $\mathrm{TiO}_{2} / \mathrm{MWCNT}$ ratio equal to 5/95 show the best results for the $\mathrm{A}_{\mathrm{ea}}$ from 48 up to 120 hours.

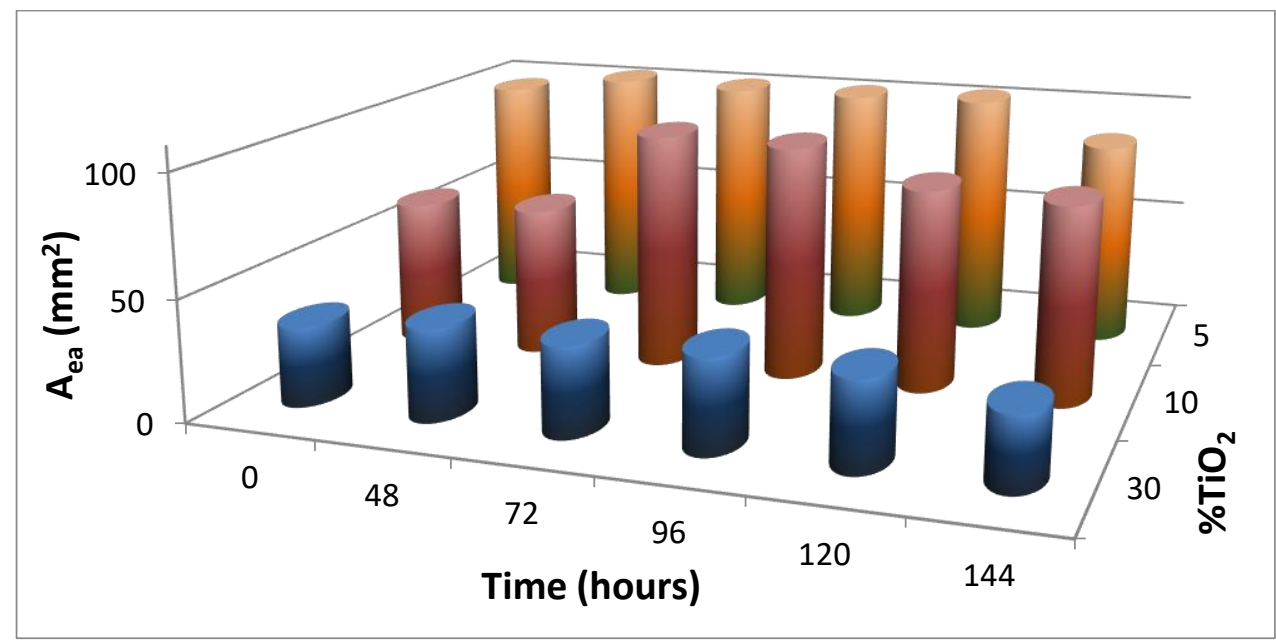

Figure 4: Stability over time of the GC/MWCNT/TiO $/[\mathrm{Ch}][\mathrm{Phe}] /$ water electrodes; $\mathrm{TiO}_{2} / \mathrm{MWCNT}$ ratios: $5: 95,10: 90$ e $30: 70 ; 1.3 \mathrm{mg}$ total mass 


\subsection{Alcohol dehydrogenase biosensor}

Alcohol dehydrogenase enzyme (ADH) to construct enzymatic biosensors has been widely used to determine ethyl alcohol level in foods [19,28 ].

The here proposed platform has been utilized, to construct a second generation sensor based on $\mathrm{ADH}$ enzyme and $\mathrm{NAD}^{+}$coenzyme as a redox mediator.

Enzyme and coenzyme were combined on the working electrode surface and to ensure the proper $\mathrm{pH}$ for the enzymatic reaction, a buffer solution $(\mathrm{pH}=8)$ instead of water, was used for RTIL solution preparation. A quantity of $40 \mathrm{IU}$ of ADH enzyme from Saccharomyces cerevisiae and the proper concentration of $\mathrm{NAD}^{+}$, was immobilized on the modified electrode surface to determine the ethyl alcohol amount in beverages. The final enzymatic platform

GC/MWCNT/TiO $/[\mathrm{Ch}][\mathrm{Phe}] /$ buffer/ADH-NAD ${ }^{+}$was then used to construct the calibration curve for ethanol analyses (Figure 5).

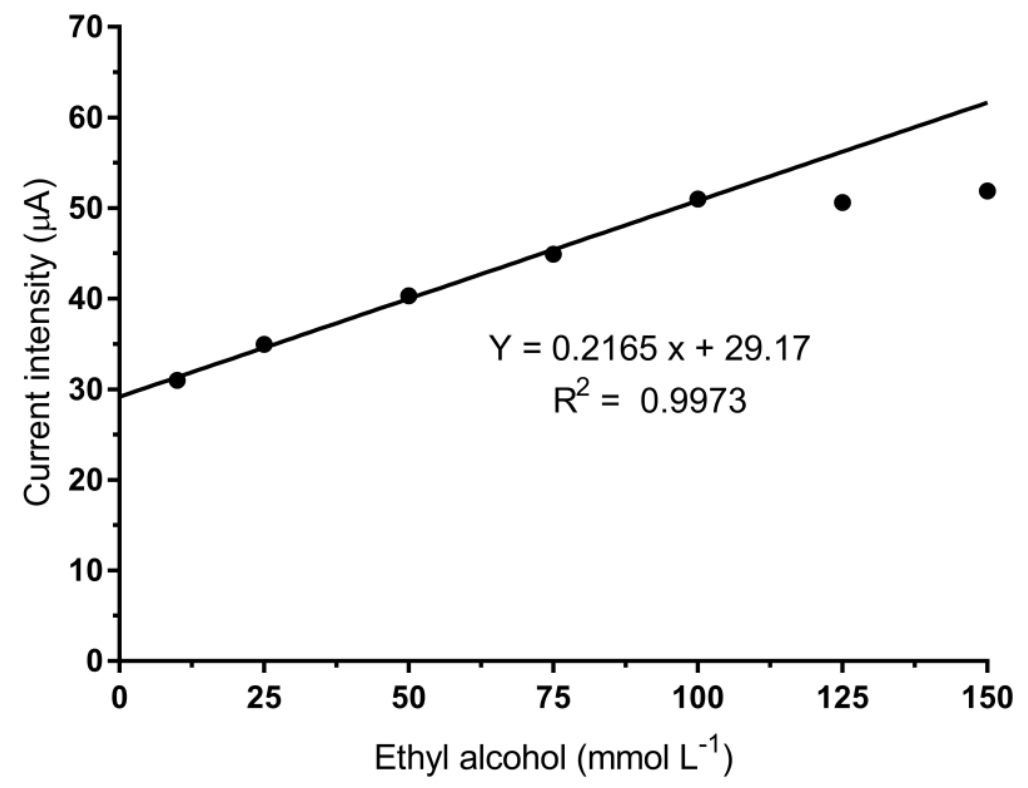

Figure 5: Calibration curve for ethyl alcohol analysis. Working electrode: GC/MWCNT/TiO $/\left[\right.$ Ch][Phe]/buffer/ADH-NAD ${ }^{+}\left(40 \mathrm{IU}\right.$ ADH-NAD ${ }^{+}$)

The analytical performances of the ADH biosensor are listed in Table 3.

\begin{tabular}{|c|c|c|c|c|}
\hline Slope & $\mathbf{R}^{\mathbf{2}}$ & LOD $\left(\mathrm{mol} \mathrm{L}^{-1}\right)$ & LOQ $\left(\mathrm{mol} \mathrm{L}^{-1}\right)$ & Linearity range $\left(\mathrm{mol} \mathrm{L}^{-1}\right)$ \\
\hline 216.49 & 0.9973 & 0,007 & 0,022 & $0.022-0.1$ \\
\hline
\end{tabular}

Table 3 Analytical performances of the ADH biosensor. Working electrode: GC/MWCNT/TiO $2 /[\mathrm{Ch}][\mathrm{Phe}] /$ buffer/ADH-NAD ${ }^{+}$

As an example of the application of the proposed biosensor to real samples, the ethanol percentage on an aquavit sample was measured. The real sample has been analyzed without any preliminary treatment, only a dilution in buffer solution was necessary. For the aquavit beverage, a hundred times dilution was used, showing that the proposed biosensor can be easily applied to very small quantities of the sample without any handling procedure. 
The found percentage of ethyl alcohol $(51.1 \pm 0.1 \%)$ has been compared with the value $(49.03 \%)$ obtained by the AOAC densimetric method, based on the hydrostatic balance $[29,30]$. The biosensor, densimetric and declared (50\%) values are in good agreement. The analysis has been performed on a distilled beverage, owing to the fact that the reference method involved a preliminary distillation of the alcoholic beverages.

\subsection{Lipase biosensor}

As already described, the lipase biosensors are based on the antioxidant compounds released from the oil matrix due to the lipolytic action on triglycerides $[6,13]$. The here proposed platform (GC/MWCNT/[Ch][Phe]/buffer) has been employed to assemble a more reliable sensor to obtain an edible oils classification. Several factors can affect the oil complex matrix, thus the categorization can be performed on the basis of different parameters. The first distinction is between olive oils and different seeds oils. Regarding the olive oils (EVOOs), the classification could be made on the basis of the various olive plant cultivar, and/or on the basis of the different geographical areas where the olive trees originate from.

Knowing that the optimal temperature for free Candida Rugosa lipase is $37^{\circ} \mathrm{C}$, the results obtained with the previously modified platform (without the $\mathrm{TiO}_{2}$ ) operating in two different ways: at room temperature $\left(25^{\circ} \mathrm{C}\right)$ with immobilized enzyme (biosensor) or at $37{ }^{\circ} \mathrm{C}$ in a batch reactor with free enzyme in solution (sensor) have been compared.

The seven EVOOs (Table 1) were analyzed using the two systems. Results are reported in Figure 6.
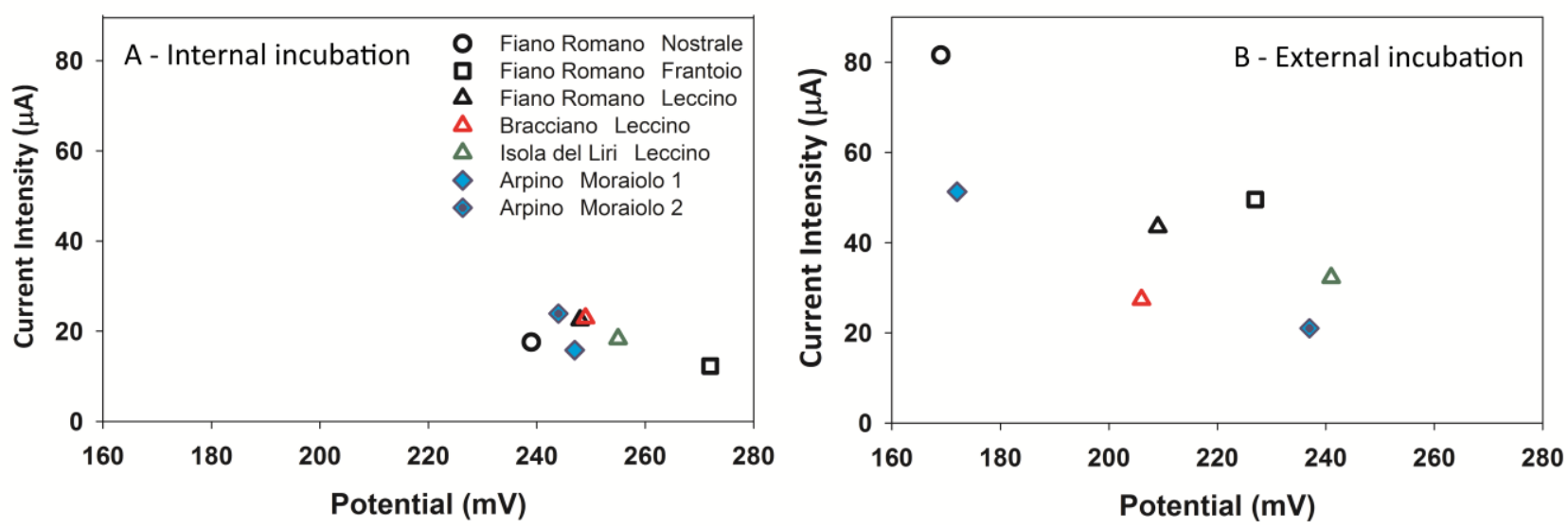

Figure 6 Electrochemical responses for oils of different cultivar and geographical origin in the presence of

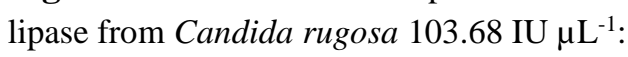

A) Biosensor based on GSI modified electrode containing lipase enzyme,

B) Sensor based on GSI modified electrode using the external enzymatic incubation

As it can be seen the best electrochemical results are obtained when the platform used as a sensor, with external enzymatic hydrolysis. In the incubator at $37^{\circ} \mathrm{C}$ the free enzyme can work in optimal conditions and it has the best access to the oil-water interface. The intensity values of the sensor results are about five times larger than those obtained for the biosensor. Relating to the possible classification of oil samples on the basis of the cultivar or the geographical origin, no significant differences are shown. It can be noted that the sensor potential and intensity values lie in a greater range with respect to the biosensor ones. Thus, external incubation has been used for all the following measures. 
The patform modified with $\mathrm{TiO}_{2}$ (see section 3.1) has been used to find out the best performances regarding olive oil analysis, obtained by $\mathrm{CV}$ measurements. In Figure 7 the $\mathrm{CV}$ of three olive oils are reported as an example.

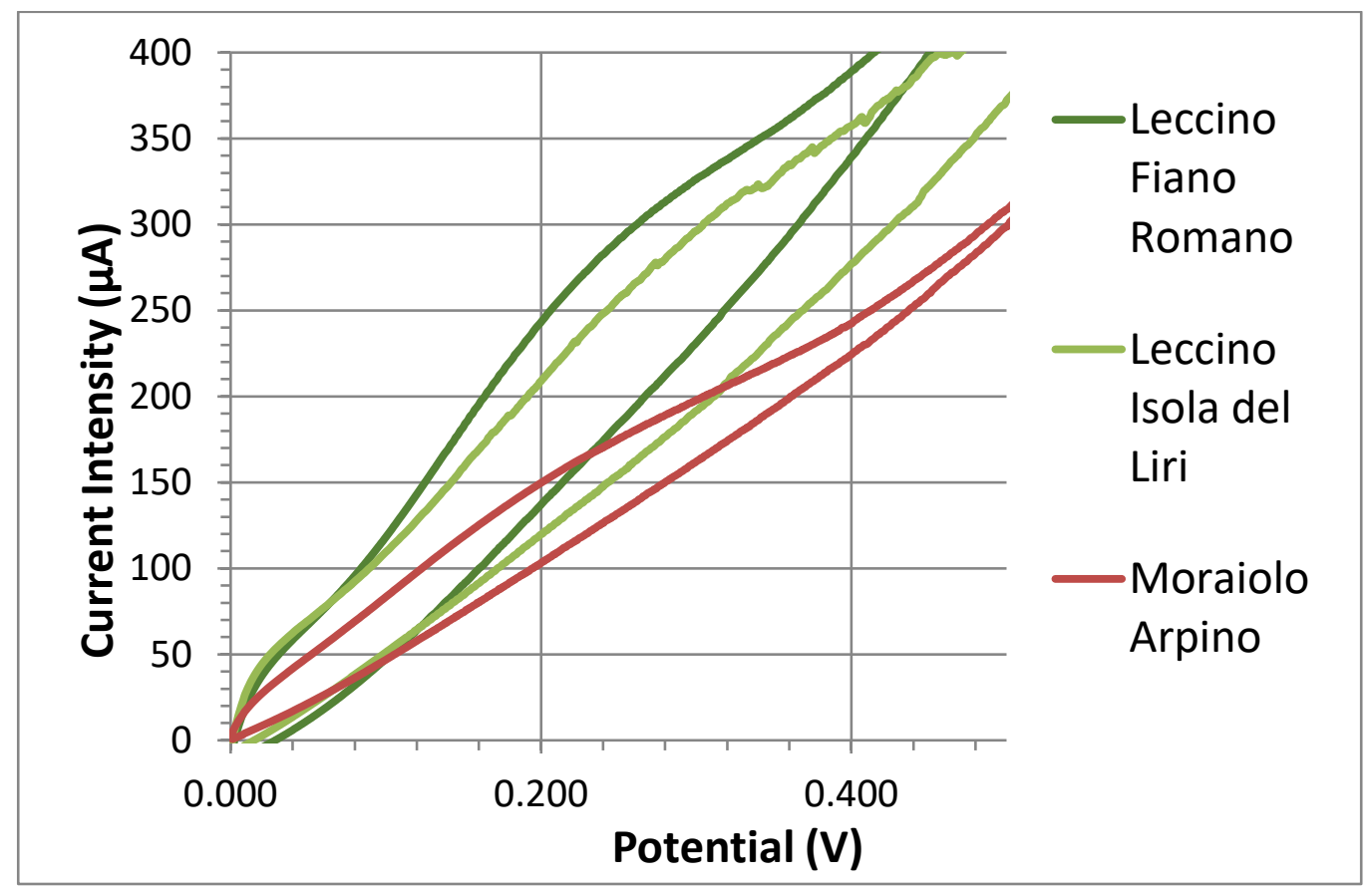

Figure 7: CV scans relative to three EVOOs (sample emulsion EVOO:[Ch][Ser]:buffer 2:2:1) performed with the GC/MWCNT/ $\mathrm{TiO}_{2} /[\mathrm{Ch}][\mathrm{Phe}]$ platform after lipase hydrolysis

In Table 4 the comparison of the results has been reported.

\begin{tabular}{|c|c|c|c|c|c|}
\hline \multirow{2}{*}{$\begin{array}{c}\text { Geographical } \\
\text { origin }\end{array}$} & \multirow{2}{*}{ Cultivar } & \multicolumn{2}{|c|}{$\begin{array}{c}\text { Oxidation potential } \\
(\mathbf{m V} \pm \text { SD) }\end{array}$} & \multicolumn{2}{c|}{$\begin{array}{c}\text { Peak current intensity } \\
(\boldsymbol{\mu A} \pm \text { SD) }\end{array}$} \\
\cline { 3 - 6 } & & MWCNT & MWCNT+TiO 2 & MWCNT & ${\mathrm{MWCNT}+\mathrm{TiO}_{2}}$ \\
\hline Fiano Romano (RM) & Nostrale & $169 \pm 1$ & $235 \pm 2$ & $81.61 \pm 0.03$ & $19.36 \pm 0.09$ \\
\hline Fiano Romano(RM) & Frantoio & $227 \pm 3$ & $224 \pm 5$ & $49.56 \pm 0.07$ & $35.30 \pm 0.05$ \\
\hline Fiano Romano (RM) & Leccino & $209 \pm 1$ & $245 \pm 2$ & $43.51 \pm 0.08$ & $35.37 \pm 0.06$ \\
\hline Bracciano (RM) & Leccino & $206 \pm 5$ & $252 \pm 3$ & $27.36 \pm 0.04$ & $26.71 \pm 0.04$ \\
\hline Isola del Liri (FR) & Leccino & $241 \pm 4$ & $243 \pm 2$ & $32.23 \pm 0.02$ & $33.30 \pm 0.02$ \\
\hline Arpino (FR) & Moraiolo 1 & $172 \pm 2$ & $202 \pm 3$ & $51.30 \pm 0.02$ & $25.03 \pm 0.03$ \\
\hline Arpino (FR) & Moraiolo 2 & $237 \pm 2$ & $258 \pm 2$ & $20.99 \pm 0.03$ & $29.28 \pm 0.03$ \\
\hline
\end{tabular}

Table 4 Comparison of the results obtained by the two platforms, without and with $\mathrm{TiO}_{2}$, for the different examined EVOOs with external enzymatic incubation

The results obtained with the $\mathrm{GC} / \mathrm{MWCNT} / \mathrm{TiO}_{2} /[\mathrm{Ch}][\mathrm{Phe}]$ platform show a good aggregation of results for the Leccino cultivar. The results regarding the other cultivars are more dispersed, which underline a possible classification for olive oil based on cultivar.

The described platform GC/MWCNT/TiO $/[\mathrm{Ch}][\mathrm{Phe}] /$ buffer has been used to analyze some seeds oils, always by means of the external lipase catalysis. In Figure 8, the results are for both seed oils and EVOOs. 


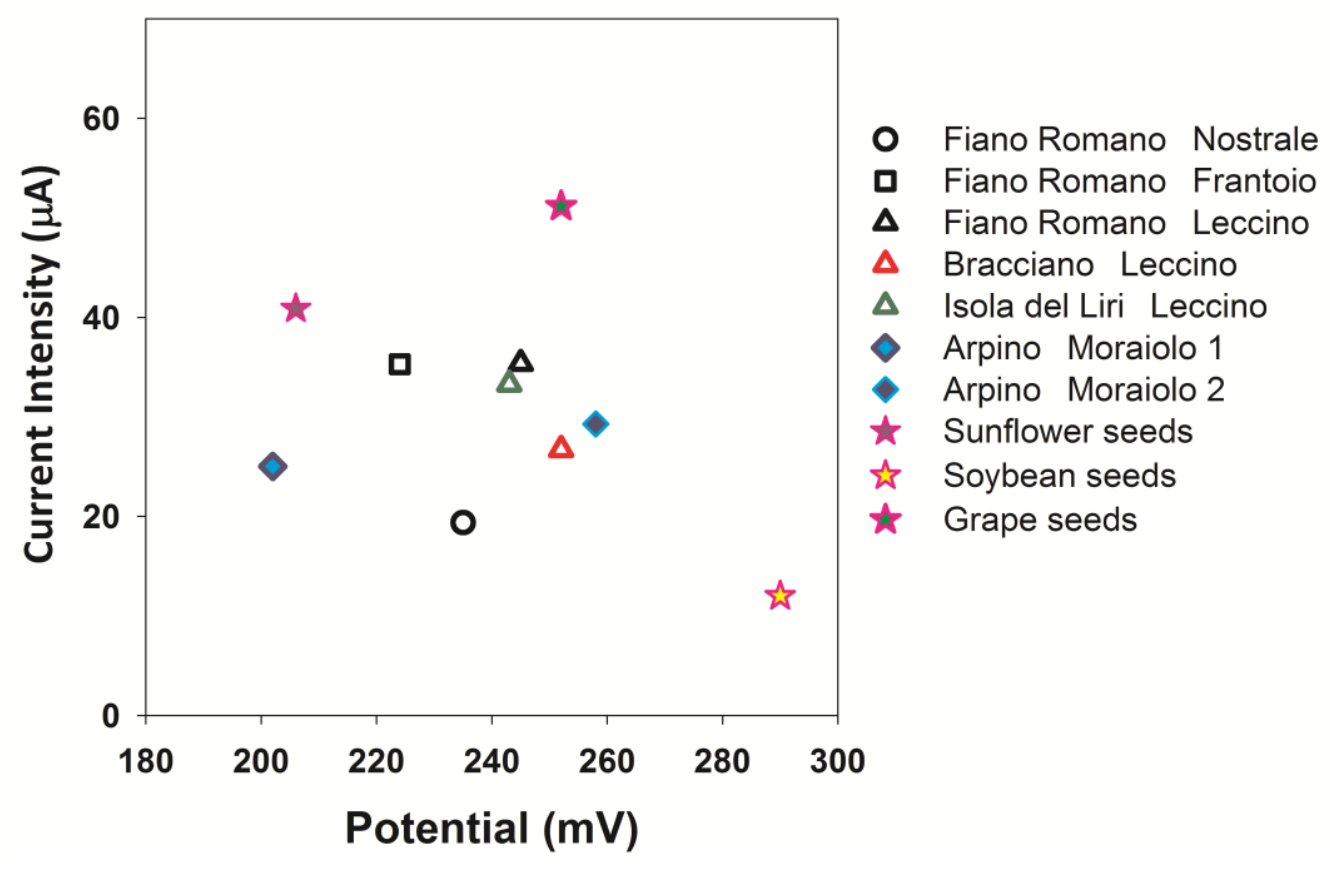

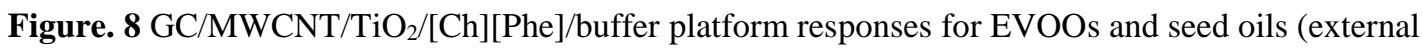
enzymatic incubation with lipase from Candida rugosa $103.68 \mathrm{IU}_{\mu} \mathrm{L}^{-1}$ )

The seed oils values compared with EVOOs ones are quite dispersed in the graph. Their behavior with respect to the antioxidants released during the triglyceride hydrolysis, resulted quite strange. Even if the commercial seed oils contained the same added amount of tocopherol $\left(0,6 \mathrm{mg} \mathrm{L}^{-1}\right)$, as stated by the manufacturer on the label, the electrochemical responses in terms of potentials and current intensities, are very different. This is probably due to the variable antioxidant composition, which is very difficult to know in the manufacturer label. In particular, for example, the high response of the grape seeds oil may be due to the high content of phenols such as oligomeric proanthocyanidins [31]. It has shown that the antioxidants power of proanthocyanidins is 20 times greater than vitamin E [32] and it could probably mask the answer due to the added vitamin E.

\section{Conclusions}

The proposed platform, obtained by modifying the WE with two nanomaterials ( MWCNT and $\mathrm{TiO}_{2}$ nanoparticles) combined with a new generation of RTIL choline based, is characterized by an $\mathrm{A}_{\text {ea }}$ grater than that shown by the preceding platform without $\mathrm{TiO}_{2}$. Consequently a higher electrochemical response is produced determining a high sensitivity of the sensors or biosensors constructed with such modified electrodes.

Being the enzyme biosensors widely used in the analytical applications [33], to analyze in a sensible and specific way samples coming from various fields, the use of RTIL choline and amino acids based, that are absolutely green allows preparing devices actually biologically friendly.

The changes made on the carbon-based electrodes allow biosensors to be constructed. A good immobilization of the enzymes is performed by the RTIL through the interactions established with the proteins, without affecting their structure and catalytic capacity.

The results obtained with the proposed platform show potential capabilities to discriminate EVOOs of different cultivar and distinguish EVOOs from non-olive oils. Other factors depending on the geographical position of the plants may affect the production of olives, so that a distinction of EVOOs based on geographical origin could emergedid not appear so evident because the distance 
between the various plants examined does not exceed $100 \mathrm{~km}$ and, as such, all of them lie in the same geographical area.

The use of these sensors and biosensors is easy handling, especially because the real samples can be analyzed without any preliminary treatment, sometimes only the dilution in buffer could be requested. The use of disposable SPEs makes these devices particularly suitable for screening and field analyses.

Conflicts of interest: none.

\section{Acknowledgments}

This work was supported by the Italian Education Ministry (MIUR) Protocol number: RM11715C53850E54 titled: "Sviluppo di metodi di screening basati su elettrodi enzimatici modificati da liquidi ionici come biosensori amperometrici e analisi di dati di raggi $\mathrm{X}$ in fluorescenza e diffrazione, rivolti alla classificazione ed al controllo della qualità degli oli di oliva". 


\section{References}

[1] A. Hasan, Md Nurunnabi, M. Morshed, P. Arghya, A. Polini, T. Kuila, M. Al Hariri, Yongkyu Lee, A. A. Jaffa, Recent advances in application of biosensors in tissue engineering, BioMed. Res. Int. (2014) Article ID 307519, 18 pages.

[2] R. Pauliukaite, A. P. Doherty, K. D. Murnaghan, C. M. A. Brett, Application of some room temperature ionic liquids in development of biosensors at carbon film electrodes, Electroanalysis 20 (2008) 485-490.

[3] K.K. Subraya, A. Diggs, D.M. Porterfield, Amperometric biosensor approaches for quantification of indole 3-acetic acid in plant stress responses, Commun. Soil Sci. Plant Anal. 44 (2013) 1749-1763.

[4] J. Bao, C. Hou, M. Chen, J. Li, D. Huo, M. Yang, X. Luo, Y. Lei, Plant esterasechitosan/gold nanoparticles-graphene nanosheet composite-based biosensor for the ultrasensitive detection of organophosphate pesticides, J. Agric. Food Chem. 63 (2015) 10319-10326.

[5] C. Tortolini, P. Bollella, M.L. Antonelli, R. Antiochia, F. Mazzei, G. Favero, DNA based biosensors for $\mathrm{Hg}^{2+}$ determination by polythymine-methylene blue modified electrodes, Biosens. Bioelectron. 67 (2015) 524-531.

[6] D. Zappi, R. Caminiti, G.M. Ingo, C. Sadun, C. Tortolini, M.L. Antonelli, Biologically friendly room temperature ionic liquids and nanomaterials for the development of innovative enzymatic biosensors, Talanta 175 (2017) 566-572.

[7] Y. Liu, M. Wang, Z. Li, H. Liu, P. He, J. Li, Preparation of Porous

Aminopropylsilsesquioxane by a Nonhydrolytic Sol-Gel Method in Ionic Liquid Solvent, Langmuir 21 (2005) 1618-1622.

[8] Z. Li, H. Liu, Y. Liu, P. He, J. Li, A Room-Temperature Ionic-Liquid-Templated ProtonConducting Gelatinous Electrolyte, J. Phys. Chem. B 108 (2004) 17512-17518.

[9] X. Lu, J. Zhou, Y. Zhao, Y. Qiu, J. Li, Room Temperature Ionic Liquid Based Polystyrene Nanofibers with Superhydrophobicity and Conductivity Produced by Electrospinning, Chem. Mater. 20 (2008) 3420-3424.

[10] H. Liu, Y. Liu, J. Li, Ionic liquids in surface electrochemistry, Phys. Chem. Chem. Phys. 12 (2010) 1685-1697.

[11] X. Lu, J. Hu, X. Yao, Z. Wang, J. Li, Composite System Based on Chitosan and RoomTemperature Ionic Liquid: Direct Electrochemistry and Electrocatalysis of Hemoglobin, Biomacromolecules 7 (2006) 975-980.

[12] S. De Santis, G. Masci, F.Casciotta, R. Caminiti, E. Scarpellini, M. Campetella, L. Gontrani, Cholinium-amino acid based ionic liquids: a new method of synthesis and physico-chemical characterization, Phys. Chem. Chem. Phys. 17 (2015) 20687-20698.

[13] R. Pauliukaite, A.P. Doherty, K.D. Murnaghan, C.M.A. Brett, Application of room temperature ionic liquids to the development of electrochemical lipase biosensing systems for water-insoluble analytes, J. Electroanal. Chem. 656 (2011) 96-101.

[14] J. S. Xu, G.C. Zhao, A Third-Generation Biosensor Based on the Enzyme-Like Activity of Cytochrome c on a Room Temperature Ionic Liquid and Gold Nanoparticles Composite Film, Int. J. Electrochem. Sci. 3 (2008) 519 - 527.

[15] Y. Zheng, Z. Liu, Y. Jing, J. Li, H. Zhan, An acetylcholinesterase biosensor based on ionic liquid functionalized graphene-gelatin-modified electrode for sensitive detection of pesticides, Sensors and Actuators B: Chemical 210 (2015) 389-397.

[16] R.Leardi, Experimental design in chemistry: a tutorial, Anal. Chim. Acta 652 (2009) 161-72. 
[17] R. Leardi, Experimental design, in: F. Marini (Ed), Chemometrics in food chemistry, Elsevier, Oxford, 2013 pp. 9-53.

[18] J.K. Park, H.J. Yee, K.S. Lee, W.Y. Lee, M.C. Shin, T.H. Kim, S.R. Kim, Determination of breath alcohol using a differential-type amperometric biosensor based on alcohol dehydrogenase, Analytica Chimica Acta 390 (1999) 83-91.

[19] F. Salimi, M. Negahdary, G. Mazaheri, H. Akbari-Dastjerdi, Y. Ghanbari-Kakavandi, S. Javadi, S.H. Inanloo, M. Mirhashemi-route, M.H. Shokoohnia, A. Sayad, A novel Alcohol Biosensor Based on Alcohol Dehydrogenase and Modified electrode with $\mathrm{ZrO}_{2}$ Nanoparticles, Int. J. Electrochem. Sci. 7 (2012) 7225 - 7234.

[20] E.J. Herrera-López, Lipase and Phospholipase Biosensors: a Review, in: Lipases and Phospholipases. Methods in Molecular Biology (Methods and Protocols), G. Sandoval (eds) Humana Press, 2012, vol 861.

[21] G. A. Alvarez, J. A. Rodriguez, J. C. Escamilla, C. A. Galan, Monoenymatic Lipase Potentiometric Biosensor for the Food Analysis Based on a pH sensitive Graphite-epoxy composite as transducer, J. Mex. Chem. Soc. 59B (2015) 19-23.

[22] E. Mariotti, M. Mascini, Determination of extra virgin olive oil acidity by FIA-titration, Food Chemistry 73(2) (2001) 235-238.

[23] EU REGULATIONS. 1991. On the characteristics of olive oil and olive-oil residue oil and on the relevant methods of analysis. CEE 2568/91: N.L.

[24] D. Zappi, G. Masci, C. Sadun, C. Tortolini, M. L. Antonelli, P. Bollella, Evaluation of new cholinium-amino acids based room temperature ionic liquids (RTILs) as immobilization matrix for electrochemical biosensor development: proof-of-concept with Trametes Versicolor laccase, Microchemical Journal 141 (2018) 346-352. doi:10.1016/j.microc.2018.05.045.

[25] M. Pacios, M. del Valle, J. Bartroli, M.J. Esplandiu, Journal of Electroanalytical Chemistry 619 (2008) 117-124.

[26] Y. Yao, G. Li, S. Ciston, R. M. Leuptow, K. A. Grey, Photoreactive $\mathrm{TiO}_{2} /$ Carbon Nanotube Composites: Synthesis and Reactivity, Environ. Sci. Technol. 42 (13) (2008), 4952-4957.

[27] S. Kang, Z. Cui, J. Mu, Composite of Carboxyl-Modified Multi-walled Carbon Nanotubes and $\mathrm{TiO}_{2}$ Nanoparticles: Preparation and Photocatalytic Activity, Fullerenes, Nanotubes, Carbon Nanostruct. 15 (2) (2007) 81-88.

[28] Ş. Alpat, A. Telefoncu, Development of an Alcohol Dehydrogenase Biosensor for Ethanol Determination with Toluidine Blue O Covalently Attached to a Cellulose Acetate Modified Electrode, Sensors 10(1) (2010) 748-764.

[29] M. L. Wang, Y. M. Choong, N. W. Su and M. H. Lee, A Rapid Method for Determination of Ethanol in Alcoholic Beverages Using Capillary Gas Chromatography, Journal of Food and Drug Analysis 11 (2) (2003) 133-140.

[30] AOAC Official Method of Analysis. 1990. Wines. In Official Methods of Analysis of AOAC International. 15th edition, pp. 739-750.

[31] J. Shi, J. Yu, J. E. Pohorly, Y. Kakuda, Polyphenolics in Grape Seeds-Biochemistry and Functionality, J Med Food 6 (4) (2003) 291-299.

[32] S. Uchida, Condensed tannins scavenging active oxygen radicals, Med. Sci. Res. 15 (1980) 831-832.

[33] P. Bollella, L. Gorton, Review Article. Enzyme-based amperometric biosensors, Current opinion in Electrochemistry (2018). https:// doi.org/ 10.1016/ j.coelec.2018.06.003. 